\title{
Computational simulation including interpretation of the importance of the immune system in tumor growth
}

\author{
Nidhi S Vaishnaw ${ }^{1} \&$ S. Shankar Narayan ${ }^{2}$
}

1,2 Department of Mathematics, CMR Institute of Technology, Bengaluru, India

Shankar.n@cmrit.ac.in ${ }^{1} \&$ Nidhi.v@cmrit.ac.in ${ }^{2}$

Article History:Received:11 January 2021; Accepted: 27 February 2021; Published online: 5 April 2021 Abstract: The secret to tumor growth and survival is proliferation. Therefore, to plan and formulate a required therapy process,
the study of the propagation rate is very important. The objective of the immune system is to protect against disease or other
potentially harmful foreign bodies, a set of processes and mechanisms within the body. In regulating the growth of a tumor,
different cells of our immune system perform their assigned function. Natural Killer cells, Dendritic cells, and CD8+ cells are
among the many cells. In our scientific literature, in the presence of numerous family components of the human immune system,
we have developed a mathematical model to evaluate the dynamics involved in tumor formation. We separated the population of
tumor cells into proliferating and inactive subsets and felt that the cells of the immune system had little effect on the dormant
cells. This partition of the universal tumor set into the subsets as mentioned has never been made in any of the previous research
works. Also, to the best of authors' knowledge, the compartment combination considered for the present work adds novelty to the
article.

\begin{tabular}{lllllllll}
\hline Keywords: & Proliferation, & Quiescent, & Natural & killer & cells, & Dendritic & cells, & CD8+ \\
\hline
\end{tabular}

\section{INTRODUCTION}

The role of the immune system in controlling cancer development, both mathematically and clinically, means that mathematical models that integrate tumor growth and treatment would do well to include a portion of the immune system. It is also possible to model how various immune therapies, once the variable is in operation, will influence the system in combination with each other or one by one. The latest clinical evidence presented and applied by Wheeler et al.[1] showed that paired with traditional cancer therapy, the capacity of the immune response has important benefits. In tandem with chemotherapy, it has been found that vaccine therapy makes outpatient survival periods more successful than either chemotherapy or vaccine therapy alone. As a result, the resulting statistical oncology continues to be a fundamental area for developments in medical therapy. It assures the inclusion of tools by precisely structured statistical formulas for both quantifying success indicators from patient-specific scientific data and tailoring cancer care. In the past few years, mathematical oncology has progressed into a complex project, advancing at a phenomenal pace.

One of the key facts of oncology statistical modeling is the simulation of the various oncological treatments used by the different forms of cancer. Model for surgery way of treatment for various cancers has been made by Hanin et al. [2] and Enderling et al. [3] Farrar et al.[4] developed a mathematical model to study the significance of the immune system in fighting cancer. The chemotherapy model was developed by several teams. Powathil et al. [5] investigated a model for chemotherapy and radiation therapy for brain tumors. The team considered a simple Spatio-temporal model to include the effect of both radiation and chemotherapeutic treatments.

It is known that persons with weakened immune systems are more likely to develop certain rare forms of cancer, particularly those with AIDS. This event can be viewed as providing more proof that the role of the immune response in the battle against cancer is crucial. See, for example, Dalgleish and O'Byrne [6]. A two-compartment tumor cell model consisting only of proliferating and quiescent cells were proposed by Gyllenberg and Webb [7]. They believed that dead cells will be automatically eliminated from the tumor, which is in reality, incorrect. Their major contribution is their theory that in a normal multicellular avascular tumor spheroid, proliferating cells may travel into and out of sleep.

Many mathematical models have suggested that the absence of the immune system leads to the growth of the tumor [8].Studies have figured out that the Natural Killer cells and cytotoxic T cells are responsible for killing the tumor cells.Natural killer cells and cells CD8+T are responded to tumor cells with high cytolytic behavior as per Osadaet al.[9]. Natural killer cells are part of the innate immune system, although there are no tumor cells. Roitt et al.,[10] noticed enabled tumor-specific CD8+T cells are found only in substantial numbers while tumor cells are present as part of the immediate immune response; natural killer and CD8+T cells are transformed into inactive cells after multiple tumors encounter[11].

In our previous study, we emphasized the importance of dead cell density in the case of the dormant stage of the tumor. As a continuation of our previous study, we model the immune system with a growing population of tumor cells. We describe the interaction between four main cell populations namely Tumor cells $T(t)$,Natural 
killing cells $N(t)$, Dendritic cells $D(t)$, and Cytotoxic $\mathrm{CD}^{+}$cells $L(t)$.This article comprises four sections. In section 2, we explore the role of immune cells in anti-tumor activity theoretically, and then a suitable mathematical model is constructed to portray the theoretical learning. Section 3 presents the mathematical analysis of the model constructed- stability has been discussed. In section 4, we analyze the results obtained by solving the system formulated in the previous section using numerical techniques, the Runge Kutta method. In the final section, we present the advantages and disadvantages of the model formed with future developments possible.

\section{A. MATHEMATiCal MODEL}

Specific prelude lymphocytes in the same family as $\mathrm{T}$ cells and $\mathrm{B}$ cells are natural killed (NK) cells. NK cells however react rapidly to a wide variety of pathological complications as endogenous immune system cells. NK cells are better positioned to cope with contaminated cells and to detect cancer early indications. First, it was noticed that NK cells could destroy or activate leading tumor cells. NK cells often contain cytokines, which are used to enhance the immune response against certain immune cells, for example, macrophages and dendritic cells. As the NK cells are in direct communication with other cells, whether or not the NK cells destroy them depends on the compatibility of the signals of actively receiving cells and the inhibitory receptors of the NK cell surface. Receptor receptors recognize and disable molecules released on the surface of cancer and contaminated cells.

Dendritic cells (DC) are a diverse, heterogeneous grouping of the tumor invading innate immune cells. DC plays an essential part in the immunity recovery of anti-tumor $\mathrm{T}$ cells and thus is one of cancer immunotherapy's main therapy strategies.

CD8 + $\mathrm{T}$ cells are another group of cells involved in tumor cell combat. For immune reaction to and control of intracellular diseases, including viruses and bacteria, the cytotoxic $\mathrm{T}$ lymphocytes or CD8 + cells are extremely important. A CD8+T cell develops three significant pathways for the destruction of damaged or diseased cells in appreciation of the antigen. In addition to the major immune protection roles of CD8+ $\mathrm{T}$ cells against pathogens, intracellular bacteria, and tumors, defective immune responses that may lead to immune disturbances and immunemediated harm are also possible.

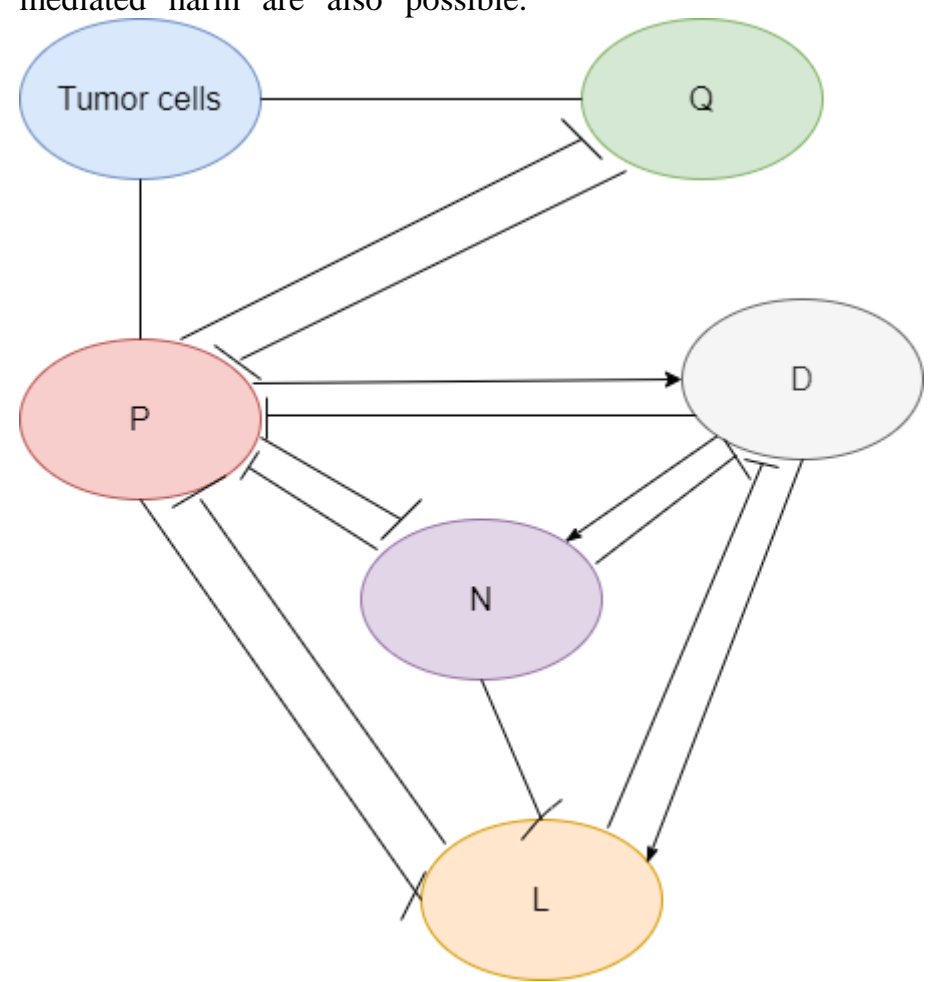

Figure 1. Block diagram representing the model constructed

Many of the above-mentioned cells are the activity in vitro effectors for various tumors. However, tumor or cellassociated inhibitory signals impede theirs in vivo efficacy. If dormancy cells are not affected by NK, DC, and 
CD8+, we devise the model below to determine how time-dependent the cell densities are. Using all of the aforementioned premises, we are formulating the following nonlinear ordinary differential equation scheme. Figure 1 represents the compartmental model for the model constructed.

$$
\begin{aligned}
& \qquad \frac{d P}{d t}=f(r) P-g(r) P+\alpha f(r) R-\left(C_{1} N+j D+k L\right) P \\
& \frac{d R}{d x}=g(r) P-\alpha f(r) R-\mu R \\
& \frac{d N}{d t}=S_{1}-C_{2} N P+d_{11} \frac{D N}{m_{1}+D}-e N \\
& \frac{d D}{d t}=S_{2}-\frac{l D P}{m_{2}+P}-f_{1} L D-d_{2} D N-g D \\
& \frac{d L}{d t}=f_{22} \frac{L P}{m_{3}+P}-h L P-i L \\
& \text { Where } P(0)=P_{0}>0, \mathbf{R}(0)=R_{0} \geq 0, \\
& f(r)=\frac{k r}{a r+1} \operatorname{andg}(r)=\frac{c}{r+m}
\end{aligned}
$$

Here $\mathrm{P}(\mathrm{t})$ and $\mathrm{R}(\mathrm{t})$ represents the proliferation and dormant cell densities respectively, $\mathrm{r}(\mathrm{t})$ plays the role of proliferating cells' limiting nutrient uptake rate function, $f(r)$ and $g(r)$ are the rates of proliferation and rate of transition from proliferation to dormancy state which is assumed to be fixed, $\alpha$ is the rate of transition of dormant cells to proliferating phase, $\mu$ is the rate of death of quiescent population, transmission between immune cells namely Tumor cells $T(t)$,Natural killing cells $N(t)$, Dendritic cells $D(t)$, and Cytotoxic CD8 ${ }^{+}$cells $L(t)$ is presented further. The values of the various parameters involved in the model are mentioned in the upcoming table 1 .

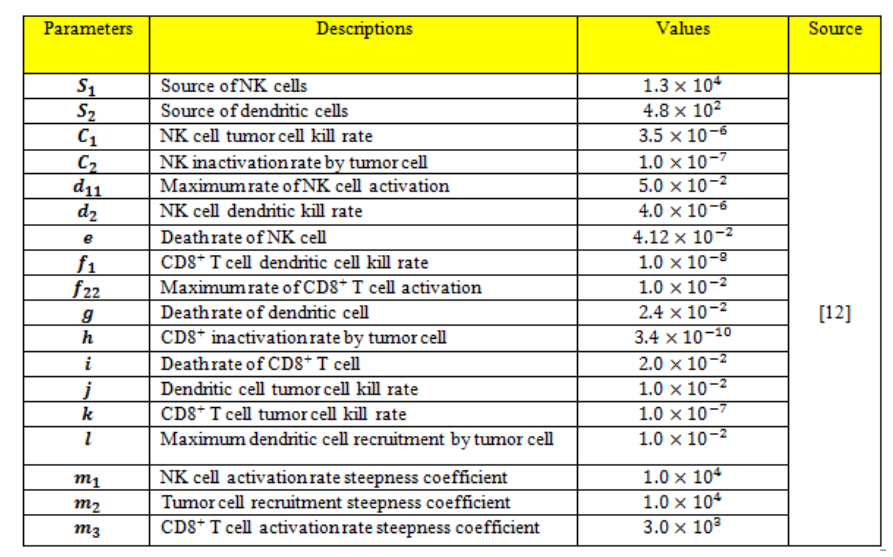

Table 1. The parameter values of the model

B. Stability Analysis \& Equilibrium points

In this section, we apply mathematical interpretation to determine tumor-free equilibrium which will identify the conditions to eliminate tumor cells.

Let $T_{0}\left(P^{*}, R^{*}, N^{*}, D^{*}, L^{*}\right)$ be a tumor-free equilibrium of the governed system(1). For an equilibrium point, we can put $\frac{d P}{d t}=\frac{d R}{d t}=\frac{d L}{d t}=\frac{d N}{d t}=\frac{d D}{d t}=0$.

On solving system (1) we get two tumor-free equilibrium as $T_{1}\left(0,0, N_{11}^{*}, D^{*}, 0\right) \& T_{2}\left(0,0, N_{12}^{*}, D^{*}, 0\right)$ 
Research Article

Where $D^{*}=\frac{s_{1}}{\left(d_{2} N^{*}+g\right)}$ and by setting $\frac{d N}{d t}=0$ leads to a quadratic equation given

$N_{1,2}^{*}=$

$\underline{\left[\left(d_{11}-e\right) S_{2}+m_{1} d_{1} s_{1}-m_{1} g e\right] \pm \sqrt{\left[\left(d_{11}-e\right) S_{2}+m_{1} d_{1} S_{1}-m_{1} g e\right]^{2}+4 e m_{1} d_{1} S_{1}\left(S_{2}+m_{1} g\right)}}$

$2 e m_{1} d_{1}$

Both tumor-free equilibrium

points are

biologically meaningful if $\left(d_{2} N^{*}+g\right)>0$

$I=\left[\begin{array}{llll}j_{11} & j_{12} & j_{13} & j_{14} j_{15} \\ j_{21} & j_{22} & j_{23} & j_{24} j_{25} \\ j_{31} & j_{32} & j_{33} & j_{34} j_{35} \\ j_{41} & j_{42} & j_{43} & j_{44} j_{45} \\ j_{51} & j_{52} & j_{53} & j_{54} j_{55}\end{array}\right]$

Where

$j_{11}=f(r)-g(r)-\left(C_{1} N^{*}+j D^{*}+k L^{*}\right)$,

$j_{21}=g(r), j_{31}=-C_{2} N^{*}, j_{41}=-\frac{I D^{*} m_{2}}{\left(m_{2}+P\right)^{2}} j_{51}=f_{2} \frac{L^{*} m_{3}}{\left(m_{2}+P\right)^{2}}-h L^{*} \quad j_{12}=\alpha f(r), j_{22}=-\alpha f(r)-\mu, j_{32}=0, j_{42}=0, j_{52}=0$

$j_{13}=-C_{1} P^{*}, j_{23}=0, \quad j_{33}=-C_{2} P^{*}+d_{11} \frac{D^{*}}{m_{1}+D^{*}}-e, j_{43}=-d_{2} D^{*}$

$j_{53}=0$

$j_{14}=-j P^{*}, j_{24}=0, j_{34}=d_{11} \frac{N^{*} m_{1}}{\left(m_{1}+D^{*}\right)^{2}}, j_{44}=-\frac{l P^{*}}{m_{2}+P^{*}}-f_{1} L^{*}-d_{2} N^{*}-g, j_{54}=f_{22} \frac{P^{*}}{m_{2}+P^{*}}$

$j_{15}=-\boldsymbol{k} \boldsymbol{P}^{*}, j_{25}=0, j_{35}=0, j_{45}=-\boldsymbol{f}_{1} \boldsymbol{D}^{*}, j_{55}=-\boldsymbol{h} \boldsymbol{P}^{*}-\boldsymbol{i}$

$I^{*}=\left[\begin{array}{ccrrrr}f(r)-g(r)-\left(C_{1} N^{*}+j D^{*}\right) & a f(r) & \text { The Tumor-free equilibrium } \\ g(r) & -a f(r)-\mu & 0 & 0 & 0 \\ -C_{2} N^{*} & 0 & d_{11} \frac{D^{*}}{m_{1}+D^{*}}-e & d_{11} \frac{N^{*} m_{1}}{\left(m_{1}+D^{*}\right)^{2}}-f_{1} D^{*} \\ -\frac{l D^{*} m_{2}}{\left(m_{2}+P\right)^{2}} & 0 & -d_{2} D^{*} & & -d_{2} N^{*}-g & -i \\ 0 & 0 & 0 & 0 & 0\end{array}\right]$

Then the Characteristic Equation of the above matrix is

given by

$$
\operatorname{det}\left[\begin{array}{ccccc}
|J-\lambda I|=0 & & & \\
f(r)-g(r)-\left(C_{1} N^{*}+j D^{*}\right)-\lambda & a f(r) & 0 & 0 & 0 \\
g(r) & -a f(r)-\mu-\lambda & 0 & 0 & 0 \\
-C_{2} N^{*} & 0 & d_{11} \frac{D^{*}}{m_{1}+D^{*}}-e-\lambda & d_{11} \frac{N^{*} m_{1}}{\left(m_{1}+D^{*}\right)^{2}}-f_{1} D^{*} \\
-\frac{\mid D^{*} m_{2}}{\left(m_{2}+P\right)^{2}} & 0 & -d_{2} D^{*} & -d_{2} N^{*}-g-\lambda_{-i}-\lambda
\end{array}\right]=0
$$

On expanding the characteristic equation,we get

$\left(f(r)-g(r)-\left(C_{1} N^{*}+j D^{*}\right)-\lambda\right)(-a f(r)-\mu-\lambda)\left(d_{11} \frac{D^{*}}{m_{1}+D^{*}}-e-\lambda\right)\left(-d_{2} N^{*}-g-\lambda\right)\left(-d_{2} N^{*}-g-\lambda\right)=0$

By the above equation, it is clear that most of the eigenvalues of the determinant are real and negative that implies the system is stable at the tumor-free equilibrium point.

\section{Results and Discussion}

The above-formulated system is solved using numerical methods (Fourth order Runge-Kutta Method) and the below-attached graphs are obtained.

Figure 2 represents the graphical representation of the population densities of tumor cells (Proliferating and dormant), immune system cells (NK cells, DC, and CD8+ T cells). Subsequent graphs denote the population of calls taken individually. 


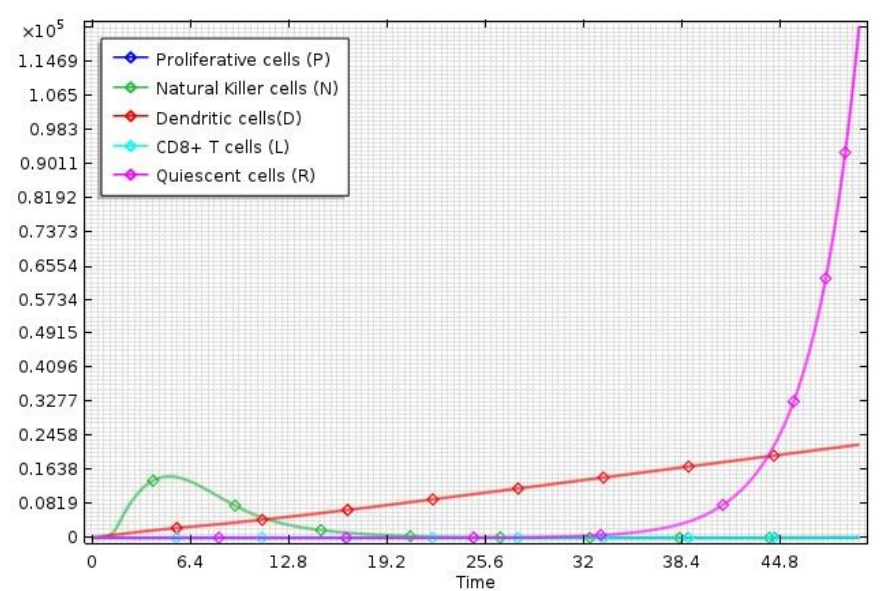

Figure 2. Population density profile of different

cells involved in the study.

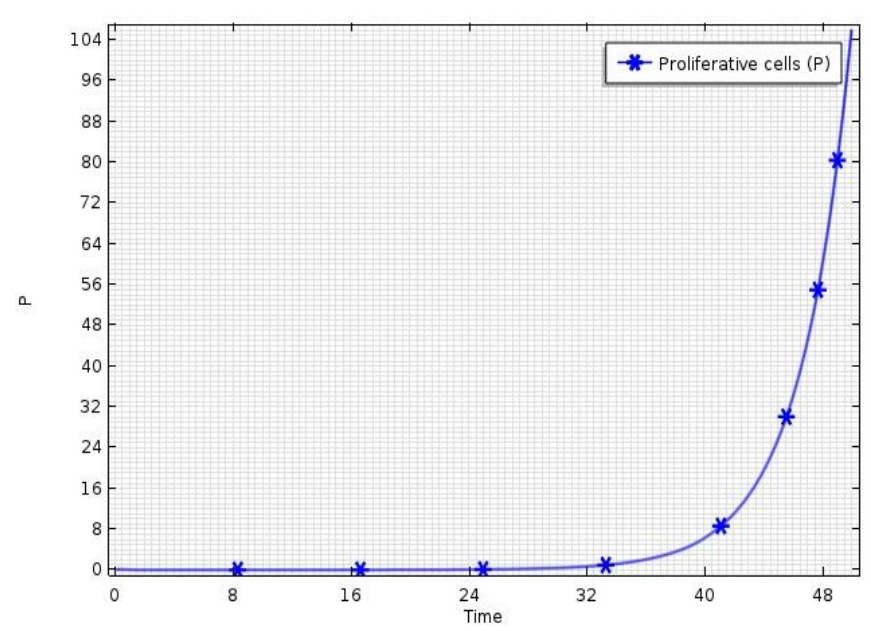

Figure 3.Population density profile of proliferative cells.

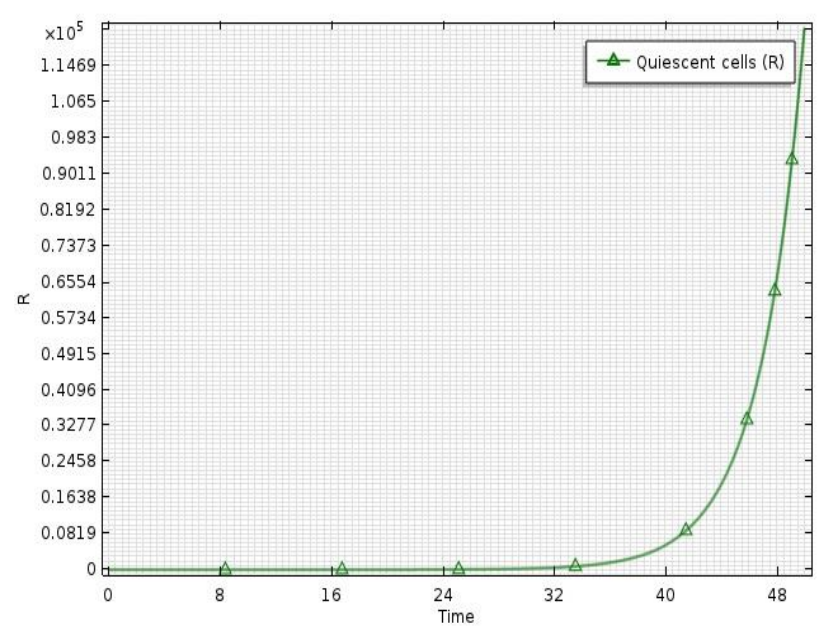

Figure 4.Population density profile of dormant cells. 


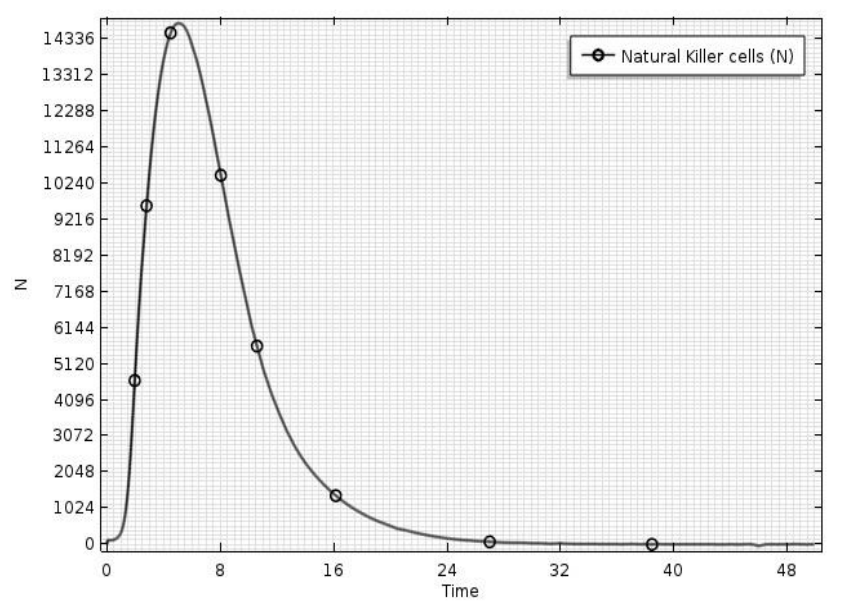

Figure 5.Population density profile of Natural Killer Cells

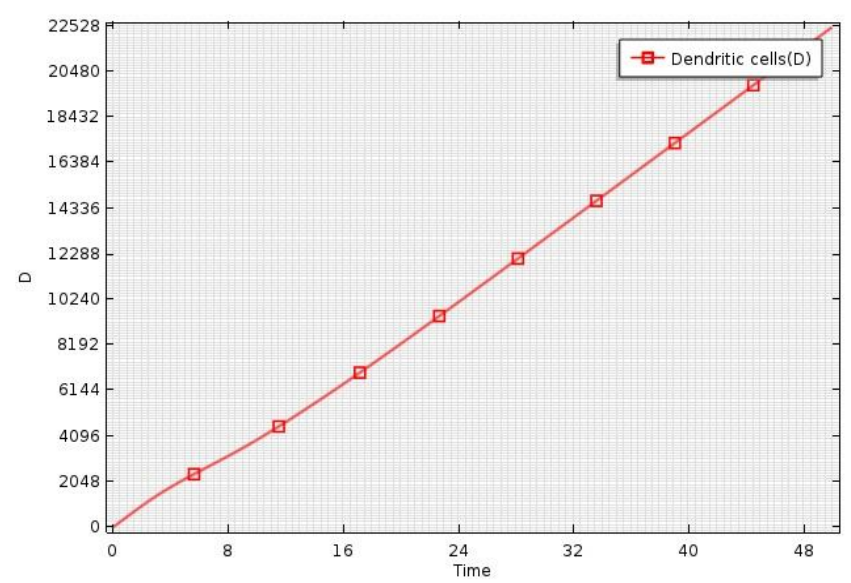

Figure 6.Population density profile of Dendritic cells population.

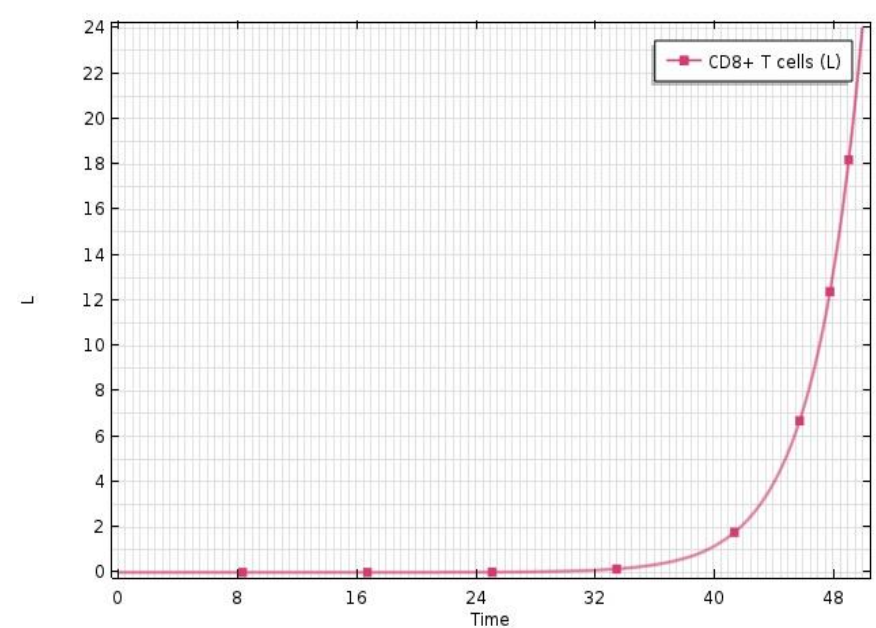

Figure 7. Population density profile of cytotoxic

The rate at which dormancy catches proliferation is high, as reported in our previous work, and it is very clear from Figures 3 and 4. It can also be shown that in the current research, the rate at which the density of proliferation declines is exceptional because immune cells are known to play an anti-tumor role responsible for 
eliminating proliferating cells from the environment. The main cause of cancer-related deaths[13] in the tumor cell process is the creation of dangerous metastatic conditions that destroy non-primary organs. The occurrence and metastasis of cancer are associated with the hibernation of cancer[14], referring to the period of prolonged idiopathy after the primary treatment of cancer. Dormancy of cancer may be considered one of the causes of adjuvant cancer which may be attributed to failure to cure original treatment of the primary tumor for malignancies[15]. As such, a basic grasp of cancer dormancy will be needed immediately to help improve cancer immunotherapy, which can be an effective alternative to metastatic cancer.

From figure 5 it is very clear that the NK cell population depletes rapidly due to its actions on the tumor target. NK cell exhaustion is an interesting aspect that is often largely ignored in NK responses to different tumors. Similar to what happens with $\mathrm{T}$ cells, the constant access to certain target antigens results in NK cells being depleted. Studies also indicated that the explanation of why the clinical result of NK cell adoption is minimal could be due to the rapid penetration ofNK cells into tumor antigens [16]. NK cells showed potent anti-tumor properties and cytokine development early on after adoption; however, NK cells exhibited poor development and cytotoxicity at the tumor sites beginning on day 5 post-transfer, figure 5. Therefore, depletion appears to be a major concern in the therapeutic use and targeting of NK cells during cancer, because rapid depletion of NK cells negatively impacts their anti-tumor functions. Repeated adoptive transfers or additional immunotherapy may be required to improve the functionality of NK cells to preserve the NK cells' strong antitumor role [16].

The dendritic cells (DC) are integral to immune system activation.DC is targeted to induce immunologic reactivity in the site of antigen expression, taking antigens, and moving into secondary lymphoid organs while receiving pathogens, dying cells, and/or cells activation signals [17]. Tumors may escape the immune function by interacting with the recruitment of DCs or by not communicating effectively. Tumors also facilitate the production of factors inhibiting DC distinction and functions. Figure 6 shows the dendritic cell density with time. The dendritic density is never exhausted even though they are deactivated by the misleading signals from the tumor cells. It has been calculated that in different species, dendritic cells are restocked from the blood at a rate of 4000 cells per hour, and experience a small number of spleen divisions over 10 to 14 days during their residence [18]. Hence the depletion is not seen in the case of the dendritic cells. But, their activity is regulated by the tumor cells. Hence immune therapy that can reactivate these dormant dendritic cells has to be employed to overcome the false signals imparted by the naughty cancer cells.

Easy CD8 + T-cell activation requires the connection to cells presenting a competent antigen, mainly with mature dendritic cells. This will increase the number of cells that attack the target and that may then be scanned for antigen-positive somatic cells across the whole body [19].From figure 7, it can be seen that at the beginning of the study the increase in the CD8+ cell density is slow as the activation of these cells needs the recruitment of dendritic cells. As time increases steadily, the number of cytotoxic cells also increases in number gradually.

\section{Conclusion}

In the present work, we formulated a mathematical model for cancer growth in presence of various immune cells that fight back these fast-dividing cells of the tumor. We validated the results obtained by comparing them with various Oncologies oriented research papers. We have investigated the role of each immune cell in combating against the tumor cell entities and misleading characteristic features of the cancer cells in inactivating these immune cells. The study clearly shows the need for various therapies to improve the efficiency of the immune cells against their inactivating agents. Hence immunotherapy is very much necessary for improving the immune system functionality to oppose proliferation. Now in the next upcoming works of our crew, we would concentrate on the different therapies available or necessary for an oncologist to employ.

The model formulated doesn't involve the entire crew of human immune system. Thus, the model could be developed to analyze the proliferation-dormancy rate by including the other immune cells of our immune system, which play a prominent role in anti-tumor activity. Also, we assumed that the rate of proliferation and the rate of transition from proliferation to dormant state was assumed to be fixed during the model construction which is not valid for the practical situations. Hence we pretend to construct a model in which the rate is a variable quantity. 


\section{References}

[1] Wheeler C. J., Asha D., Getao L., Yu J.S., Black K. L., " Clinical responsiveness of glioblastoma multiforme to chemotherapy after vaccination”, Clin. Cancer Res.,10, pp 5316-5326, 2004.

[2] Hanin L, Seidel K, StoevesandtD ,A "universal" model of metastatic cancer, its parametric forms and their identification: what can be learned from site-specific volumes of metastases Leonid Hanin, Karen Seidel \& Dietrich Stoevesandt. J Math Biol 72, pp 1633-1662,2015.

[3] Enderling H, Anderson AR, Chaplain MA, Munro AJ, Vaidya JS ,Mathematical modelling of radiotherapy strategies for early breast cancer. J Theor Biol 241, pp 158-171,2005.

[4] Alfonso J, Jagiella N, Núñez L, Herrero MA, DrasdoD ,Estimating dose painting effects in radiotherapy: a mathematical model. PLoS ONE 9:e89380,2014.

[5] PowathilG, KohandelM,SivaloganathanS, OzaA,MilosevicM,Mathematical modeling of brain tumors: effects of radiotherapy and chemotherapy. Phys Med Biol 52,pp 3291-3306(2007).

[6] Dalgleish A. G., O' Byre K.J, , Chronic immune activation and inflammation in the pathogenesis of AIDS and cancer, Adv. Cancer Res. 84, pp 231-276,2002.

[7] Gyllenberg M, Webb GF: Quiescence as an explanation of Gompertzian tumor growth. Growth Dev Aging, 53,pp 25-33, 1989.

[8] Britton N. F., Essential Mathematical Biology, Springer, Berlin.

[9] Osada, T., Nagawa, H., Shibata, Y., Tumor-infiltrating effector cells of a-galactosylceramide-induced antitumor immunity in metastatic liver tumor. J. Immune. Based Ther. Vaccines 2 (7),pp1-9,2004.

[10] Roitt, I.M., Brostoff, J., Male, D.K.,. Immunology. Mosby, St.Louis 1993.

[11] Yang Kuang, John D. Nagy, Steffen E. Eikenberry, "Introduction to Mathematical Oncology", Chapman and Hall/CRC Mathematical and Computational Biology, 2015,series,p. 86-90, 2015.

[12] T. Trisilowati, S. McCue, and D. Mallet, "Numerical solution of an optimal control model of dendritic cell treatment of a growing tumor," ANZIAM Journal, vol. 54, pp. 664-680,2013.

[13] Pantel K, Brakenhoff RH. Dissecting the metastatic cascade. Nat Rev Cancer. (2004) 4:448-56. $10.1038 /$ nrc 1370 .

[14] Aguirre-Ghiso JA. Models, mechanisms and clinical evidence for cancer dormancy. Nat Rev Cancer. 2007,7:834-46. 10.1038/nrc2256

[15] Goss PE, Chambers AF. Does tumor dormancy offer a therapeutic target? Nat Rev Cancer. 2010 10:871-7. $10.1038 / \mathrm{nrc} 2933$.

[16] Gill S, Vasey AE, De Souza A, Baker J, Smith AT, Kohrt HE, Florek M, Gibbs KD, Jr, Tate K, Ritchie DS, Negrin RS. Rapid development of exhaustion and down-regulation of eomesodermin limit the antitumor activity of adoptively transferred murine natural killer cells. Blood. 2012 Jun 14;119(24):5758-68.

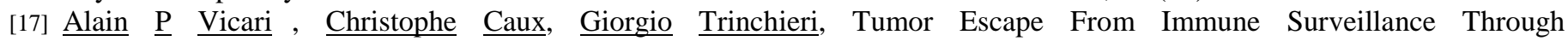
Dendritic Cell Inactivation, Semin Cancer Biol. 2002 Feb;12(1):33-42.doi: 10.1006/scbi.2001.0400.

[18] Liu, Kang; Waskow, Claudia; Liu, Xiangtao; Yao, Kaihui; Hoh, Josephine; Nussenzweig, Michel (June 2007). "Origin of dendritic cells in peripheral lymphoid organs of mice". Nature Immunology. 8 (6): 578-583. doi:10.1038/ni1462.

[19] Hivroz C, Chemin K, Tourret M, Bohineust A "Crosstalk between T lymphocytes and dendritic cells". Critical Reviews in Immunology. 32 (2): 139- 55,2012. doi:10.1615/CritRevImmunol.v32.i2.30. 